UDC $82-822$

DOI: $10.17223 / 24109266 / 10 / 15$

\title{
DIE DEUTSCHEN VERSANTHOLOGIEN DER 2. HÄLFTE DES 20. - ANFANG DES 21. JAHRHUNDERTS (LITERARISCHER ASPEKT)
}

\author{
S.Yu. Steklyannikova
}

\begin{abstract}
Zusammenfassung. Der vorliegende Artikel stellt das Ergebnis der Untersuchung der deutschsprachigen Versanthologien der 2.Hälfte des XX. - Anfang des XXI. Jahrhunderts dar. Das steigende Interesse an interkulturellen literarischen Beziehungen und hohe Aufmerksamkeit zu Anthologie seitens Herausgeber am Anfang des XXI. Jahrhunderts rücken die Anthologie in den Fokus der Literaturwissenschaft und Imagologie. Die Analysemethodik stützt sich auf die letzten anthologischen Untersuchungen der deutschen und russischen Wissenschaftler. Im Artikel wird ein Versuch unternommen, das Korpus der deutschsprachigen Anthologien zu systematisieren und statistisch zu bearbeiten. Die allgemeinen Tendenzen der deutschen Anthologisierung werden ausführlich analysiert und dargestellt.
\end{abstract}

Schlüsselwörter: deutschsprachige Anthologie, russische Lyrik, Imagologie, Rezeption, literarischer Kanon, Übersetzung.

\section{Einführung}

Im Zusammenhang mit dem wachsenden Interesse der modernen Literaturwissenschaft am Problem der interkulturellen, insbesondere interliterarischen Beziehungen, Rezeption und Imagologie, am Verständnis der Fragen des Kanons in der nationalen Literatur ist das Studium der Rezeption russischer Lyrik in deutschen Anthologien heutzutage eine aktuelle Studie. Neue Rezeptionsmaterialien der russischen Poesie auf Grund der deutschsprachigen Anthologien implizieren die Identifizierung von Strategien der Wahrnehmung der russischen Literatur in einem fremden und transkulturellen Raum, eine umfassende Aufarbeitung vielfältiger rezeptiver Tatsachen. Die Fragen der Übernahme des einen Literaturkanons in die fremde Kultur sind diskutabel: ob die anerkannten russischen Dichter genauso in einem anderen kulturellem Raum beachtet werden? Mit welchen Texten werden sie dann vorgestellt? Oder werden die Dichter mit ihren Texten, in vielen Fällen nur Übersetzungen im anderen gesellschaftlichen Feld neu interpretiert? Von diesem Standpunkt sind die deutschsprachigen Anthologien ganz wesentlich an der Vermittlung ausländischer Literatur, im weitesten Sinne am internationalen Kulturaustausch beteiligt und werden als Objekt unserer Forschung näher betrachtet. Das Korpus der Untersung besteht aus 41 deutschsprachigen Versanthologien der 2.Hälfte des XX. - Anfang des XXI. Jahrhunderts, die in Deutschland und in der Schweiz herausgegeben wurden und entweder ausschließlich oder teilweise russische Lyrik präsentieren. 


\section{Methodisches Vorgehen}

In der vaterländischen Literatur wurde die Aufmerksamkeit der Wissenschaftler in zwei letzten Jahrzehnten auf die kollektiven Sammlungen der 2.Hälfte des XX. - Anfang des XXI. Jahrhunderts [1] und auf die Definition der Anthologie gelenkt: so definierte Yu.V. Smirnowa die Anthologie als eine kollektive Sammlung der ausgewählten Werke, die die Literatur der bestimmten Periode, Richtung, eines Landes und so weiter präsentiert, und in die die wertvollsten Werke eingehen und die die einheitlichste Vorstellung davon vermittelt, was auf diesem Gebiet geschrieben wurde [2]. Die deutsche Herausgeberin Birgit Bödeker definiert die Anthologie als jedes Kompendium von Texten von mindestens drei Autoren [3: 1].

Aus dem Übersetzungsstandpunkt wurden die deutschen Anthologien des XIX. - des XX. Jahrhunderts vom Göttinger Sonderforschungsbereich "Die literarische Übersetzung" erforscht, er vereinigt die Studien im Rahmen vom Armin Paul Frank initiierten Projekt "Übersetzungsanthologien: die Etablierung von Weltliteratur: ein Paradigma der deutschen Übersetzungskultur" [3,4]. Die Wissenschaftler kommen zu dem Ergebnis, dass diese Publikationsform als der Phänomen der Kulturgeschichte im deutschen Sprachraum gelten kann. Dabei wird das umfassende Zentralkorpus mit Hilfe der von Lutz Rühling vorgestellten mathematisch - statistischen Methoden ausgewertet und die Position, das Prestige jeder Nationalliteratur durch den Vergleich der Zählungen auf der literarischen Weltkarte bestimmt.

\section{Untersuchung}

Der Göttinger Sonderforschungsbereich "Die literarische Übersetzung" hat anthologische Klassifikationen und Termini dargestellt, die hinsichtlich der Anthologien der russischen Lyrik im Artikel "Die russische Lyrik in deutschsprachigen Anthologien 2000-2010" von N. E. Nikonova und S. Y. Stekljannikova [5] weiterentwickelt wurden. Bei der Studie der ausländischen Versanthologien sind folgende Stichpunkte (Parameter) zu beachten:

1) Bilateralität/ Multilateralität wird nach der Anzahl der eingereichten nationalen Literaturen bestimmt; die bilateralen Anthologien geben einen Kommunikationsraum für zwei nationale Traditionen, die multilateralen vereinigen viele nationale Traditionen. Im Korpus der untersuchten Anthologien sind 28 bilateral, d. h sie vertreten nur russische Lyrik, 13 sind multilateral, also Weltdichtungsanthologien. Dabei ist eine Tendenz zu bemerken, dass die Weltdichtungsanthologien besonders aktiv in der Nachkriegszeit erscheinen, die letzte in dieser Periode ist die Anthologie "Chorus an die verkehrte Welt. Russische Dichtung des 18. Jahrhunderts" 1983 von Annelies und Helmut Grasshoff [6], dann nach einer langen Pause 20052006 werden sofort 3 thematische multilaterale Anthologien von Andrea 
Wüstner herausgegeben: "Himmlische Boten. Gedichte und Geschichten von Engeln, " Das Meer. Gedichte", "Die Sonnengedichte" [7-9].

2) Einsprachigkeit/ Zweisprachigkeit der Texte, die Art der Übersetzung; Anthologien werden in zweisprachige (gewöhnlich wird dabei das bekannte Ausgabeprinzip an regarde verwendet) und einsprachige Anthologien geteilt. Im letzten Fall hat der Leser eine Möglichkeit, nur die Übersetzungen wahrzunehmen. $\mathrm{Zu}$ beachten ist, dass praktisch alle multilateralen Anthologien sind einsprachig. Eine Ausnahme ist dabei nur eine Anthologie des schweizerischen Slavisten Peter Brang "Landschaft und Lyrik. Die Schweiz in Gedichten der Slaven"[10], in der es zusammen mit den deutschen auch russische, polnische, bulgarische und andere Texte gibt. Bilaterale Anthologien können sowohl zweisprachig als auch einsprachig sein. Eine sehr wichtige Rolle spielt die Strategie bei der Übersetzungsauswahl. Der poetische Text kann folgender Weise vorgestellt werden: 1) in einer bekannten, schon veröffentlichten Versübersetzung, 2) in einer neuen Versübersetzung vom Herausgeber, 3) in einer buchstäblichen Interlinearübersetzung, die in der Regel zusammen mit dem Originaltext gegeben wird. In unserem Korpus der deutschen Anthologien gehören 10 Anthologien der russischen Lyrik zum zweisprachigen Typ, 2 Anthologien "Russische Lyrik. Von den Anfängen bis zur Gegenwart" von Kay Borovsky и Ludolf Müller 1991 [11] und "Die russische Lyrik" von Bodo Zelinsky 2002 [12] haben die Interlinearübersetzung; 27 Anthologien sind einsprachige multilaterale Sammlungen, eine Anthologie gehört zum gemischten Typ: "Mein Rußland" von Karl Dedezius präsentiert nur das erste Gedicht jedes Dichters auf Deutsch und Russisch, weitere Texte nur auf Deutsch [13].

3) neue Interpretation der Schaffen der russischen Dichter; Die deutschen Anthologien schaffen eine eigene Demission der russischen Lyrik, in der die anerkannten Klassiker und marginale Dichter, die großen und die verkannten oder vergessenen Autoren von einem anderen Standpunkt gesehen werden, als es im russischen Literaturkanon der Fall ist. In diesem neuen System wird das Schaffen des einen oder anderen Dichters anders interpretiert und auf ganz neue Weise wahrgenommen. Diese Tatsache wird ausführlich in dem Artikel von Ulrike Ekutsch über übersetzerische und literarischkritische Rezeption I. I. Dmitriew erläutert [14]. Eine ähnliche Untersuchung hat die Rezeption des Schaffens von W. A. Zhukowskij betroffen. Aus dem Korpus der Anthologien wird festgestellt, dass Zhukowskij als der Dichter der Vorromantik vor dem deutschen Leser erscheint. Die Anzahl der in Anthologien angeführten Texte von Zhukowskij, die Genre und der Umfang der Texte bezeugen, dass seine Übersetzungsfertigkeiten und poetisches Erbe von den deutschen Herausgebern hoch geschätzt werden [15].

4) Der politische Faktor soll berücksichtigt werden, und zwar die Teilung Deutschlands in zwei Staaten (1949-1991). In den in der BRD und in der DDR publizierten Anthologien ist die Teilung keineswegs nur eine 
formal - staatliche, sondern sie wiederspiegelt sich in der Rezeption von den Nationalliteraturen, Autoren und Texten. Z.B. die Lyrik von W. Majakowskij wird in den westdeutschen Sammlungen durch die Gedichte vertreten, die mit dem Thema der Revolution nicht gekoppelt sind. Dagegen in den ostdeutschen Sammlungen ist er dem deutschen Leser als ein eifriger Revolutionär vorgestellt. Aus 22 Anthologien dieser Periode sind 15 in der BRD (davon 10 sind russische Sammlungen) herausgegeben, 7 - in der DDR (3 sind der russischen Lyrik gewidmet, die letzten 4 Weltdichtungsanthologien stehen im Zeichen des internationalen Sozialismus).

5) die Persönlichkeit des Herausgebers der Anthologie; Eine wichtige Rolle bei der systematischen Untersuchung der Anthologie spielt der Herausgeber, denn gerade er schafft einen Kontext, ein gewisses semantisches Feld, für dessen Übergabe er sorgfältig die poetischen Texte auswählt. In unserem Korpus sind 16 Anthologien von einer Person kommentiert und zusammengestellt, In 41 deutschen Anthologien der russischen Lyrik und der Weltdichtungsanthologien, die in der Zeitperiode von 1950 bis 2012 erschienen sind, wurden die Verfasserstrategien der Darstellung der Russischen Lyrik dem deutschen Leser untersucht. eine Typologie von Strategien des Autors zu erstellen. Die Aufgaben sind die Charakterisierung und Beschreibung der individuellen Strategien des Autors oder des Herausgebers. Im zur Verfügung stehenden Corpus der Anthologien ist möglich eine Aussonderung von 4 Typen von Herausgeber - Strategien zu machen: 1) der Autor als Vertreter der Kultur des Absenders, der authentische Sicht auf die russische Dichtung präsentiert; 3 Verfasser gehören zur Absenderkultur: E. Etkind "Russische Lyrik. Gedichte aus drei Jahrhunderten" [16], A. Nitzberg "Selbstmörder-Zirkus. Russische Gedichte der Moderne" [17] und "Sprechende Stimmen" [18], E. Lebedewa "Auf der Suche nach einem verlorenen Leid. Neue russische Literatur" [19]. Die anderen Herausgeber gehören zur deutsch - europäischen Kultur. 2) der Autor-Verfasser bietet einen innovativen Blick auf anthologische Darstellung der russischen Lyrik, richtet sich auf die Faszination und zugleich auf die transkulturelle Ebene und die Mehrsprachigkeit; A. Nitzberg "Selbstmörder-Zirkus. Russische Gedichte der Moderne" und "Sprechende Stimmen", F. Ingold "Russische Lyrik von 2000 bis 1800" [20]. 3) der Autor-Verfasser mit dem möglichst kompetenten Blick und der akademischen Installation auf die Darlegung der Russischen Lyrik; z. B., die Anthologien "Russische Lyrik. Von den Anfängen bis zur Gegenwart" von Kay Borowsky und Ludolf Müller 1991 und "Die russische Lyrik" von Bodo Zelinsky 2002 mit ausführlicher Einführung in die Entwicklung des russischen Verses, mit dem Anmerkungsteil, wo zu jedem Dichter bio-bibliographische Informationen gegeben werden, auch Wortund Sacherklärungen zu den Gedichten. Die Zielgruppe solcher Ausgaben sind Studenten der russischen Literatur. 4) der Verfasser, der die klassische Art der ausländischen Versanthologien vorstellt. Solche Anthologien mit 
unstrittigen Texten von klassischem Rang sollen dem allgemeinen Publikum in möglichst umfassender Weise die russische Lyrik präsentieren.

\section{Ergebnisse}

In der 2. Hälfte des XX. Jahrhunderts hatte "klassische" Überblicksanthologie mit ihren universellen Anspruch ausgedient. Seit etwa 1960 wählen die Anthologisten unter bestimmten Gattungen (C. Ferber «Russische Sonette aus drei Jahrhunderten" [21]), Perioden (H. Baumann "Russische Lyrik 1185-1963" [22]), Autorentypen (F. Göpfert "Das deutsche Werk Karolina Karlovna Pavlovas" [23]) oder wenden sich zu einem einzigen Thema ( G. Bodeit "Seit ich dich liebe" [24]). Statt kanonisierter Dichter mit einer fast voraussagbaren Gedichtauswahl wird Platz für jüngere, unbekannte Dichter gefunden (F. Ingold "Russische Lyrik von 2000 bis 1800" [20]). Im XXI. Jahrhundert in neuem Gewand erscheinen die Übersetzungen von älteren Werken. Noch eine markante Tendenz dieser Periode ist die Herausgeberspräferenz der bilateralen Anthologien, die dem Leser eine literarische Tradition präsentieren, die Weltdichtungsanthologien kommen sehr selten vor. Nach der Art der Vorlegung der poetischen Texte sind einsprachige Anthologien populär, in denen der Leser nur die Übersetzungen in der Muttersprache wahrnimmt. Im XXI. Jhd. werden aber auch markante bilaterale Anthologien von F. Ingold "Russische Lyrik von 2000 bis 1800" [20] und von P. Brang " Landschaft und Lyrik" [10] herausgegeben. Die Ergebnisse der Studie zeigen die vielfältigen Strategien der deutschen Verfasser in der Vorstellung der russischen Lyrik, ihr Streben eine möglichst breiten Leserkreis zu decken, die Popularität der Ausgaben solcher Art nicht nur in der DDR, sondern auch in der BRD. Die Analyse der Vorworte und Kommentare zeigt, dass bei der Auswahl der Gedichte die deutschen Anthologisten 3 Grundprinzipien in Betracht ziehen: Aufmerksamkeit auf die deutsche Literatur, die in russischer Tradition dargestellt wird, Bestreben nach dem Konstruieren des möglichst vollen Bildes der russischen Lyrik und die thematische Auswahl.

\section{Literaturverzeichnis}

1. Bazhenova, V.V.: Russkiy literaturnyy sbornik serediny XX-nachala XXI veka kak tseloe: al'manakh, antologiya [Russian analects of the middle of the 19th - early 21 st centuries as a whole: almanacs, anthologies]. Abstract of Philology cand. diss. Nivosibirsk. (2010). (In Russian)

2. Smirnova, Yu.V.: Antologiya kak raznovidnost' poeticheskogo sbornika [Antology as a type of poetic collection]. Abstract of Philology cand. diss. Moscow. (2003). (In Russian)

3. Boedeker, B., Essman, H.: Einleitung // Weltliteratur in deutschen Versanthologien des 20. Jahrhunderts. Berlin: Erich Schmidt Verlag. (1997). (In German)

4. Essman, H., Schoening, U.: Weltliteratur in deutschen Versanthologien des 19. Jahrhunderts. Berlin: Erich Schmidt Verlag. (1996). (In German) 
5. Nikonova, N.E., Steklyannikva, S.Yu.: Russkaya poeziya v nemetskikh antologiyakh 20002010-kh gg.: dinamika izdatel'skikh strategiy i chitatel'skikh ozhidaniy [Russian poetry in the German athologies of the 2000s-2010s: dynamics of publishing strategies and reader expectations]. Text.Book.Publishing. 1(10). pp.138-154. (2016). (In Russian)

6. Grasshoff, H., Grasshoff, A.: Chorus an die verkehrte Welt. Russische Dichtung des 18. Jahrhunderts. Philipp Reclam Jun.Leipzig. (1983). (In German)

7. Wüstner, A.: Himmlische Boten. Gedichte und Geschichten von Engeln. Philipp Reclam Jun. Stuttgart. (2005). (In German)

8. Wüstner, A.: Das Meer. Gedichte. Philipp Reclam Jun. Stuttgart. (2005). (In German)

9. Wüstner, A.: Die Sonne. Gedichte. Philipp Reclam Jun. Stuttgart. (2006). (In German)

10. Brang, P.: Landschaft und Lyrik. Die Schweiz in Gedichten der Slaven. Schwabe \& Co. AG. Verlag. Basel. (1998). (In German)

11. Borowsky, K., Müller, L.: Russische Lyrik. Von den Anfängen bis zur Gegenwart [Russian lyric. From the beginnings to the present]. Philipp Reclam Jun. Stuttgart. (1983). (In German)

12. Zelinsky, B., Herlth, J.: Die russische Lyrik [Russian lyric]. Köln, Weimar, Wien: Böhlau. (2002). (In German)

13. Dedecius, K.: Mein Rußland in Gedichten [My Russia in poems]. Deutscher Taschenbuch Verlag GmbH \& Co. KG, München. (2003). (In German)

14. Ekuch, U.: Dmitriev v Germanii. K voprosu o perevodcheskoy i literaturno-kriticheskoy retseptsii [Dmitriev in Germany. On the question of translation and critical reception]. St. Petersburg: RAS. pp.129-145. (2010). (In Russian)

15. Steklyannikova, S.Yu.: Poeziya V.A. Zhukovskogo v nemetskih antologiyah XX-XXI vv. [Poetry of V.A. Zhukovsky in German anthologies of XX-XXI centuries]. Actual problems of literary criticism and linguistics: collections of materials II (XVII) International Conference of Young Scientists (April 18-23, 2016). Tomsk: Publishing house of Tomsk State University. 17. pp. 311-315. (2016). (In Russian)

16. Etkind, E.: Russische Lyrik. Gedichte aus drei Jahrhunderten [Russian lyric. Poems from three centuries]. R. Piper \& Co. Verlag, München. (1981). (In German)

17. Nitzberg, A.: Selbstmörder-Zirkus. Russische Gedichte der Moderne [Suicide Circus. Modern Russian poetry]. Leipzig: Reclam Verlag Leipzig. (2003). (In German)

18. Nitzberg, A.: Sprechende Stimmen: russische Dichter lesen; Originalaufnahmen [Speaking Voices: Reading Russian poets. Original recodings]. Köln: DuMont. (2003). (In German)

19. Lebedeva, K.: Auf der Suche nach einem verlorenen Leid. Neue russische Literatur [In search of lost burdens. New Russian literature]. Edition Druckhaus. Berlin. (1994). (In German)

20. Ingold, P.P.: Als Gruss zu lesen. Russissche Lyrik von 2000 bis 1800 [To read as greeting. Russian poetry from 2000 to 1800] Dörlemann Verlag AG, Zürich. (2012). (In German)

21. Ferber, C.: Russische Sonette aus drei Jahrhunderten. Bern. (1989). (In German)

22. Baumann, H.: Russishe Lyrik 1185 - 1963. Gigbert Mohn Verlag. Gütersloh. (1963). (In German)

23. Göpfert, F.: Texte und Materialien zur russischen Frauenliteratur. Band 1.1. Das deutsche Werk Karolina Karlovna Pavlovas. Potsdam. (1994). (In German)

24. Bodeit, G.: Seit ich dich liebe. Gedichte von Frauen aus zwei Jahrhunderten. Verlag für die Frau. DDR Leipzig. (1977). (In German)

Information about the author:

Steklyannikova S.Yu. - Senior Lecturer, Derpatment of German, National Research Tomsk State University, PhD Student of philological faculty at the National Research Tomsk State University. E-mail: stekljannik@mail.ru 